\title{
Análise morfométrica e ultra-estrutural do nervo óptico de ratos induzidos a ingestão de álcool
}

\author{
Morphometric and ultrastructural analysis of the optic nerve in rats following induced \\ ethanol consumption
}

\author{
Alvio Isao Shiguematsu ${ }^{1}$ \\ Silvana Artioli Schellini \\ Elisa Aparecida Gregório \\ Cláudia Helena Pellizzon ${ }^{4}$ \\ Sérgio Swann Müller \\ Carlos Roberto Padovani ${ }^{6}$
}

\section{R E S U M O}

Objetivo: Analisar os efeitos da ingestão crônica de álcool sobre o nervo óptico em um modelo murino adulto. Métodos: Doze ratos machos da raça Wistar, de 30 dias de idade, foram divididos por sorteio em 2 grupos experimentais: "tratado" (TG), com 8 animais, alimentados com raçãopadrão para roedores de laboratório e uma mistura de água de torneira e etanol ad libitum; "controle" (CG), com 4 animais, alimentados com a mesma ração e água de torneira pura ad libitum. Após 40 semanas todos os ratos foram sacrificados, sendo os nervos ópticos de ambos os olhos preparados para microscopia óptica e eletrônica. A área de secção transversal de cada nervo a aumento de $500 \times$, assim como número de fibras axonais dentro de 5 campos aleatoriamente selecionados a aumento de $2000 \times$ foram medidos com auxílio de digitalizador de imagens acoplado ao microscópio óptico. Foram realizadas fotomicrografias de 10 campos aleatoriamente selecionados de cada nervo ( 5 centrais e 5 periféricos) a aumento de $4200 \times$ em microscópio eletrônico de transmissão. Resultados: A análise morfométrica não mostrou diferenças estatisticamente significativas entre os 2 grupos estudados. Em contraste com o CG, o exame ultra-estrutural dos nervos ópticos do TG mostrou um intenso desarranjo das bainhas de mielina, que se tornaram espessadas, com separação de suas lamelas, apresentando, por vezes, degenerações interlamelares elétrondensas, além da presença de muitas organelas degeneradas. Conclusão: Os achados desse estudo mostram alterações ultra-estruturais no nervo óptico de ratos adultos após ingestão crônica de álcool, sem modificações morfométricas significativas.

Descritores:Nervoóptico/citologia;Nenoóptico/ultraestrutura;Microscopiaeletrônica; Etanol/toxidade;Ratos

I N T R O D U Ç ̃̃ O

Aproximadamente 2/3 dos americanos acima de 14 anos consomem bebidas alcoólicas. Etilistas "pesados" constituem cerca de $10 \%$ destes (7\% da população adulta dos Estados Unidos) e respondem por aproximadamente metade do consumo de álcool e pela quase totalidade das complicações sócio-econômicas e médicas do etilismo e abuso de álcool. O custo anual destes problemas para a sociedade americana é de cerca de 100 bilhões de dólares ${ }^{(1)}$.

O alcoolismo crônico, como é bem sabido, induz a alterações patológicas em praticamente todos os órgãos e tecidos do organismo. Os efeitos do etanol sobre o sistema nervoso são extensos e uma variedade de desordens neuropatológicas e neuropsicológicas têm sido descritas em alcoolistas 
crônicos $^{(1)}$. O alcoolismo também está associado a alterações oculares, tais como a ambliopia por tabaco-álcool e as manifestações oculares da síndrome alcoólico-fetal $(\mathrm{SAF})^{(2)}$. Algumas alterações clínicas no sistema oculomotor também têm sido descritas, dentre elas o nistagmo ${ }^{(3)}$.

Estudos experimentais acerca dos efeitos do consumo de álcool sobre o nervo óptico de ratos têm sido mais freqüentemente limitados à exposição gestacional ${ }^{(4-6)}$ ou pós-natal pre$\operatorname{coce}^{(7-9)}$, concentrando-se principalmente na SAF. A maioria, mas não todos os estudos experimentais sobre a exposição crônica ao etanol em ratos adultos, indicam perda de células nervosas no sistema nervoso central (SNC) $)^{(8-12)}$.

O objetivo do presente estudo é analisar os efeitos da ingestão crônica de álcool sobre o nervo óptico em um modelo murino adulto por meio de análise morfométrica e ultraestrutural.

\section{MÉ T O D O S}

O projeto de estudo foi previamente aprovado pelo Comitê de Ética em Experimentação Animal da Faculdade de Medicina de Botucatu - UNESP, seguindo as normas da Association for Research in Vision and Ophthalmology (ARVO).

\section{Animais e dieta}

Doze ratos machos da raça Wistar, de 30 dias de idade, com pesos similares, foram previamente avaliados por profissional especializado quanto à ausência de doenças e então divididos, por sorteio, em 2 grupos experimentais: "tratado" (TG), com 8 animais, alimentados com ração-padrão para roedores de laboratório e uma mistura de água de torneira e etanol $a d$ libitum; "controle" (CG), com 4 animais, alimentados com a mesma ração e água de torneira pura ad libitum. Todos os animais foram mantidos em gaiolas individuais no biotério do Instituto de Biociências da UNESP - Campus de Botucatu. Foi realizado controle mensal do peso dos animais em balança eletrônica com precisão de 5 gramas.

A concentração de etanol na mistura do TG foi aumentada gradualmente de $10 \%$ na $1^{a}$ semana para $20 \%$ na $2^{a}$ semana e então para $30 \%$ até o final do experimento. Todos os animais foram mantidos em suas dietas durante 40 semanas e então sacrificados por injeção intraperitoneal de pentobarbital sódico.

\section{Preparo do tecido}

Ambos os olhos de todos os ratos foram imediatamente enucleados e seccionados ao nível do equador, imersos em uma solução de glutaraldeído $2,5 \%$ por 24 horas, sendo os nervos ópticos então dissecados e seccionados transversalmente em sua inserção no bulbo ocular. Os nervos foram então lavados em tampão de fosfato $0,1 \mathrm{M} \mathrm{e} \mathrm{imersos} \mathrm{em} \mathrm{um} \mathrm{fixador} \mathrm{secundário}$ a base de tetróxido de ósmio 1\% por mais duas horas. Após isso eles foram desidratados em uma sequiência gradual de álcool e então embebidos em resina epóxi (Araldite).

Cortes transversais semifinos $(\sim 1 \mu \mathrm{m})$ a partir da extremidade distal de cada nervo, adjacente ao bulbo, foram feitos e corados com azul de toluidina $1 \%$ para microscopia óptica de imersão. Cortes transversais ultrafinos $(\sim 80 \mathrm{~nm})$ foram então feitos usando-se um micrótomo Ultracut UCT (Leica, Áustria), sendo estes então capturados em grades de cobre (200 campos) e subseqüentemente corados com citrato de chumbo $0,2 \%$ e uma solução saturada de uranil acetato para microscopia eletrônica de transmissão.

\section{Morfometria}

As áreas de secção transversal de cada nervo, excluindo-se suas meninges, a uma magnificação de $500 \times$, assim como o número de axônios dentro de 5 campos randomizadamente selecionados por nervo, a uma magnificação de $2000 \times$, foram medidos através de um digitalizador e analisador de imagens KS 300 (Zeiss, Alemanha). Consideramos "axônios" todas as áreas circunscritas medindo entre 0,2 e $3,0 \mathrm{~mm}$, conforme descrito ${ }^{(13)}$ para a raça Wistar.

\section{Microscopia eletrônica}

Foram realizadas fotomicrografias de 10 campos ( 5 centrais e 5 periféricos) aleatoriamente selecionados de cada nervo, sem intersecções, a uma magnificação de $4200 \times$, usando-se um microscópio eletrônico de transmissão Phillips EM 301.

\section{Estatística}

Os pesos foram analisados mensalmente, usando-se o teste de Mann-Whitney. Os resultados das medidas morfométricas foram analisados através do teste $t$ de Student para amostras independentes. O nível de significância considerado para ambos os testes foi de $\mathrm{P} \leq 0,05$.

\section{RES U L T A D O S}

Não foram encontradas diferenças estatisticamente significativas entre os pesos dos ratos de TG e CG (Tabela 1) em nenhuma das 10 medidas mensais, pelo teste de Mann-Whitney.

À análise morfométrica, não foram encontradas diferenças estatisticamente significativas entre os grupos em estudo quanto às áreas de secção transversal dos nervos ópticos (Tabela 2) ou número de axônios por campo (Tabela 3), pelo teste $t$ de Student.

A análise ultraestrutural não mostrou alterações importantes em nenhum dos nervos ópticos do CG (Figura 1). Por outro lado, todos os nervos ópticos do TG apresentaram fibras axonais com muitas organelas degeneradas, além de severo desarranjo estrutural de suas bainhas de mielina, com separação de suas lamelas e, por vezes, inclusões interlamelares elétron-densas (Figura 2).

D I S C US S ÃO

O sistema nervoso é particularmente sensível aos efeitos do consumo de álcool; o nervo óptico, em especial, tem sido extensivamente estudado para determinar tais efeitos.

A análise ultraestrutural em nosso estudo mostrou sinais degenerativos severos em todos os nervos ópticos do TG, em contraste com os do CG, nos quais não foram encontradas alterações significativas. Tendo em vista a uniformidade dos achados nos 2 grupos isoladamente e o contraste marcante 


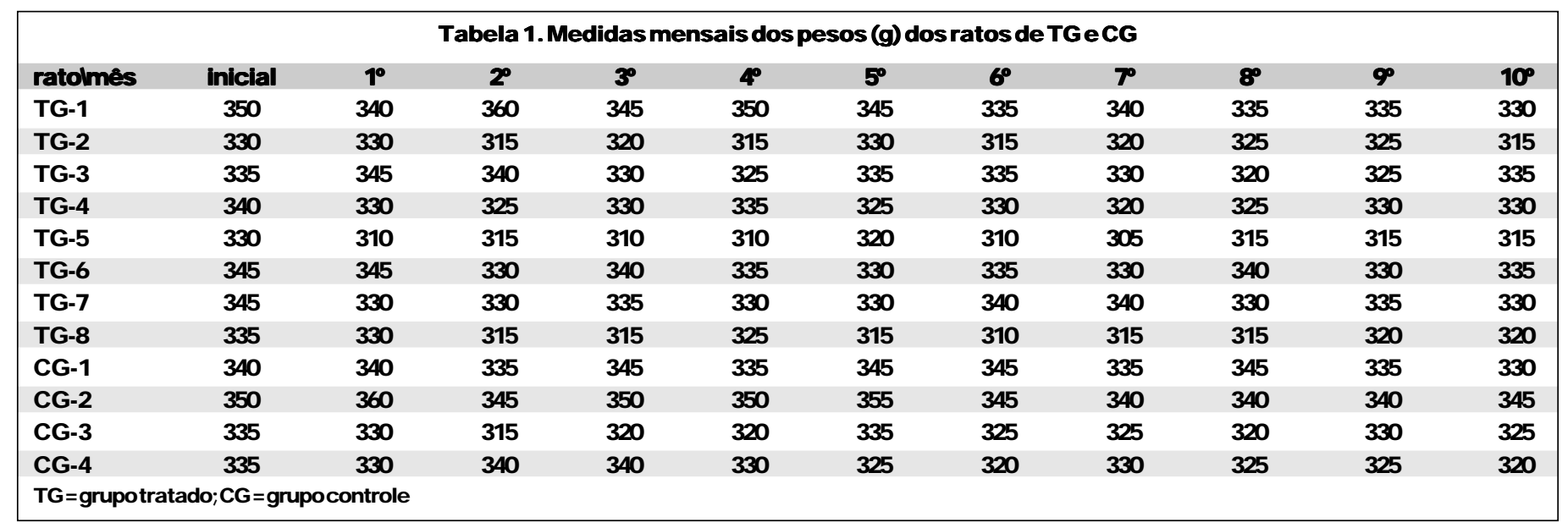

\begin{tabular}{|c|c|c|c|}
\hline Área $\left(\mathbf{m m}^{2}\right)$ & TG & CG & $\begin{array}{c}\text { Testet } \\
\text { deStudent* }\end{array}$ \\
\hline OD & $0,313 \pm 0,033$ & $0,330 \pm 0,030$ & 0,87 \\
\hline OE & $0,314 \pm 0,027$ & $0,322 \pm 0,037$ & 0,40 \\
\hline AO & $0,314 \pm 0,030$ & $0,326 \pm 0,032$ & 0,65 \\
\hline
\end{tabular}

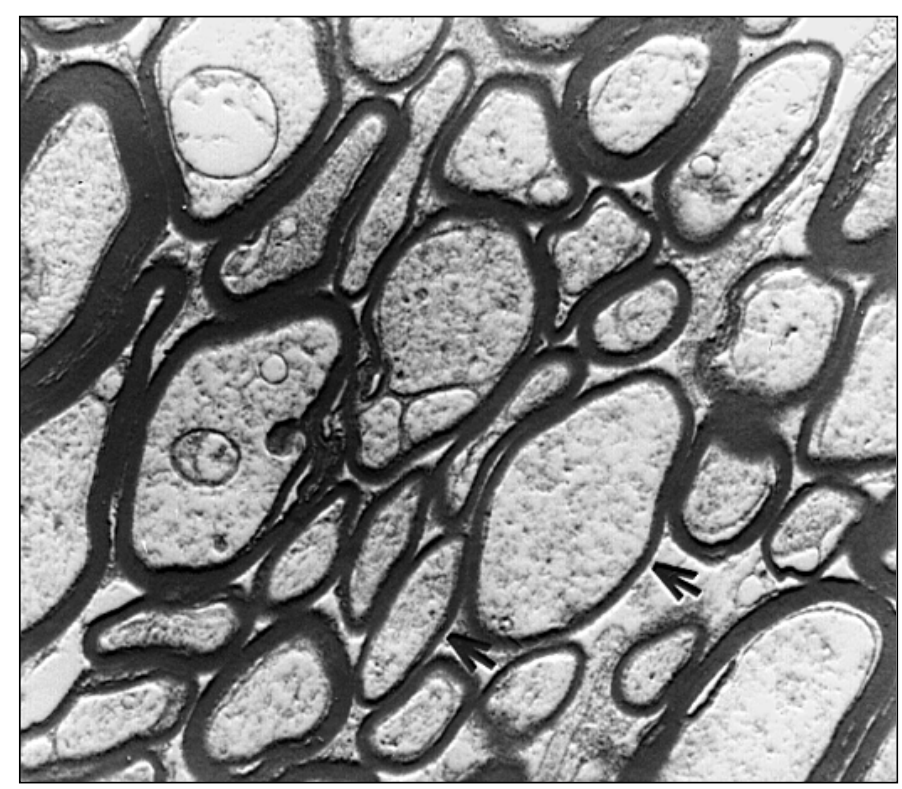

Figura 1-Cortetransversaldonenóbticodorato CG-3 examinadoao microscóploeletrônicodetransmissaáoauma magnificaçãode 4200x, evidenciandofibrasaxonaissemalteraçōes importantes (setas)

dos entre os achados ultraestruturais, consideramos suficiente o número de animais do CG.

As alterações observadas no TG sugerem contraste com estudo anterior ${ }^{(10)}$, onde não foram observados quaisquer sinais de degeneração ou alterações discerníveis em outros processos

\begin{tabular}{|c|c|c|c|}
\hline \multicolumn{4}{|c|}{ 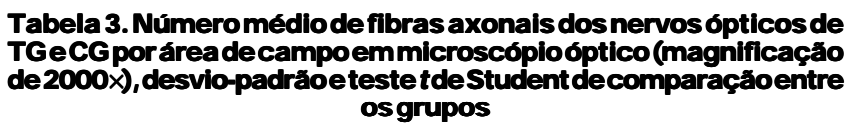 } \\
\hline №deaxônios & TG & CG & tdeStudent* \\
\hline OD & $718,4 \pm 62,5$ & $685,6 \pm 63,7$ & 0,85 \\
\hline OE & $717,0 \pm 53,1$ & $712,7 \pm 16,8$ & 0,15 \\
\hline AO & $717,7 \pm 56,9$ & $699,1 \pm 38,0$ & 0,58 \\
\hline \multicolumn{4}{|c|}{ TG $=$ grupotratado; $\mathbf{C G}=$ grupo controle. $*$ Significante para $p \leq 0,05$} \\
\hline
\end{tabular}

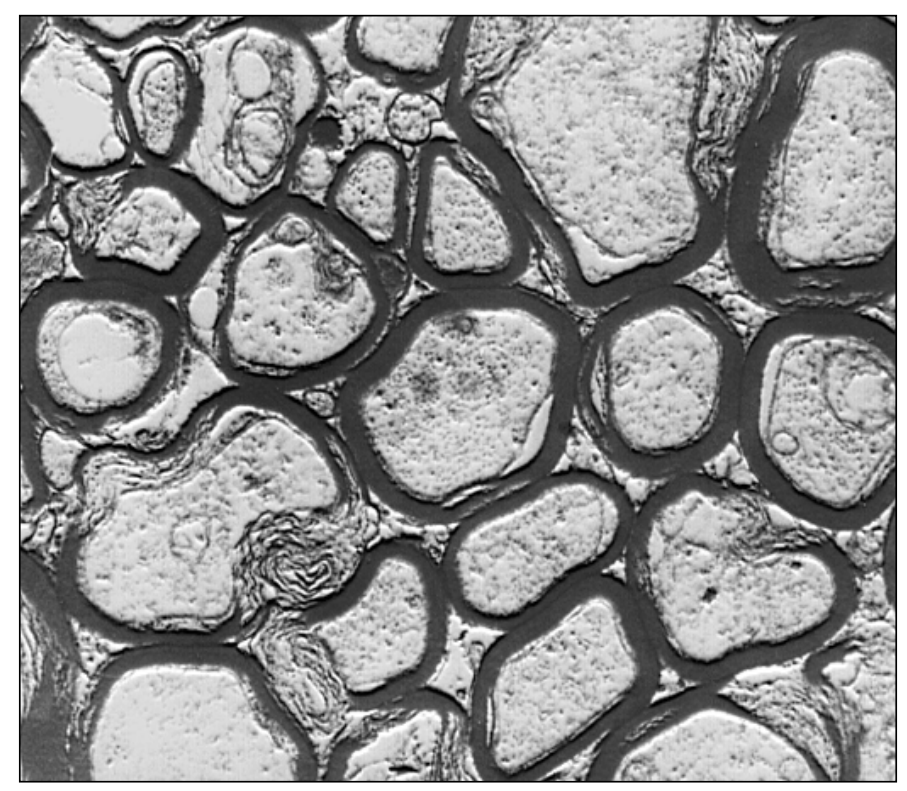

Figura 2-Cortetransversal donervó́pticodoratoTG-3examinadoao microscópioeletrônicodetransmissãoauma magnificaçãode 4200x, evidenciandofibras axonais nas quais nota-seseverodesarranjoestrutural das bainhas de mielina, comseparaçãodesuas lamelas

celulares no nervo óptico de ratos adultos alimentados com etanol 5\% por 5, 10 e 17 semanas. Em nosso estudo, porém, a concentração de etanol na solução do TG bem como o tempo de exposição foram consideravelmente maiores. Não utilizamos um 
modelo "pair-fed" (alimentados aos pares), mais próximo do ideal, onde a quantidade de calorias ingeridas pelo coelho tratado é a mesma do seu "par-controle", sendo as calorias proporcionadas pelo álcool no TG substituídas por carboidratos no CG. Contudo, a dieta ad libitum administrada em nosso estudo, mostrou-se satisfatória, tendo-se em vista que a única fonte de água dos animais do TG era a solução alcoólica (o que os obrigou a ingerir a solução) e que os pesos dos ratos não diferiram estatisticamente entre os 2 grupos durante o estudo.

Alguns autores observaram degenerações à análise ultraestrutural no SNC de ratos cronicamente alimentados com dietas contendo álcool. Degenerações induzidas pelo álcool em células hipocampais e sinapses ${ }^{(11,14-15)}$ e em células do córtex cerebelar $^{(12)}$ foram descritas.

A análise morfométrica em nosso estudo não mostrou diferenças significativas na área de secção transversal ou no número de fibras axonais entre os nervos ópticos de TG e CG. Apesar das diferenças nos métodos de tratamento por álcool e de morfometria, nossos resultados estão em concordância parcial com os de outro estudo ${ }^{(10)}$, onde se observou ausência de perda axonal após ingestão crônica de álcool em ratos adultos; entretanto nesse estudo foi notado, ao contrário do nosso, redução nas áreas de secção transversal dos nervos ópticos.

Os efeitos da ingestão crônica de álcool (2,5; 6,5; 9,5 meses) sobre células hipocampais de ratos foram anteriormente estuda$\operatorname{dos}^{(11)}$ e observou-se redução do número de células, bem como reversibilidade dos sinais degenerativos após 1 mês de abstinência. Não podemos afirmar se as alterações ultraestruturais observadas em nosso estudo são ou não reversíveis, mas morfologicamente, o grau de degeneração de muitos axônios do TG parece ser tão avançado que supomos que o dano nessas fibras seja irreversível. Como a análise morfométrica não considera a funcionalidade da fibra nervosa, é possível que muitos axônios não-funcionantes ou "mortos" tenham sido contados. Testes eletrofisiológicos e estudos envolvendo abstinência podem ser úteis para um melhor esclarecimento dessas dúvidas.

As discrepâncias entre o nosso e a maioria dos estudos podem ser devidas, em parte, às diferenças entre os procedimentos experimentais (raça, idade inicial, concentração de etanol nas dietas, tempo de exposição, preparação tissular, etc.).

\section{O N C L U S Ã O}

Os achados deste estudo mostram alterações ultraestruturais no nervo óptico de ratos adultos após ingesta crônica de álcool, sem modificações morfométricas significativas. Estudos envolvendo um modelo "pair-fed", testes eletrofisiológicos e indução de abstinência podem contribuir para um melhor esclarecimento do assunto.

\section{A B S T R A C T}

Purpose: To analyze the effects of chronic ethanol intake on the optic nerve in an adult rat model. Methods: Twelve male, 30-day-old Wistar rats were randomly divided into 2 experi- mental groups: "treated" (TG), with 8 animals, fed standard laboratory rodent chow and a mixture of tap water and ethanol ad libitum; and "control" (CG), with 4 animals, fed the same chow and pure tap water ad libitum. After 40 weeks all rats were sacrificed, having their optic nerves prepared for both licht and electron microscopy. The cross-sectional area of each nerve, at a magnification of $\times 500$, as well as the number of axons within 5 randomized field areas, at a magnification of $\times 2000$, were measured by using an image digitizer. Randomized sampling was made for taking 10 photomicrographs of each nerve ( 5 central and 5 peripheral) at a magnification of $\times 4200$ in a transmission electron microscope. Results: Morphometric analysis showed no significant differences between both groups. In contrast with CG, the ultrastructural examination of TG optic nerves showed severe disarrangement of myelin sheaths, which became thicker, with separation of their lamellae, presenting sometimes electron-dense degenerations between them, and also many degenerated organellae. Conclusion: Our findings show ultrastructural alterations in the optic nerve of adult rats following chronic ethanol consumption, without morphometric changes.

Keywords: Optic nerve/cytology; Optical nerve/ultraestructure; Microscopy, electron; Ethanol/toxity; Rats

REFERÊNCIAS

1. Robbins SL. Environmental and nutritional diseases. In: Cotran RS, Robbins SL, Kumar V, editors. Robbins pathological basis of disease. $5^{\text {th }}$ ed. Philadelphia: WB Saunders; 1994. p.388-99.

2. Jones KL, Smith DW. The fetal alcohol syndrome. Teratology 1975;12:1-10.

3. Yoshii F, Kobakake K, Shinohara Y, Takagi S. Neurological manifestations in chronic alcoholics. Tokai J Exp Clin Med 1985;10:615-20.

4. Dangata YY, Kaufman MH. Morphometric analysis of the postnatal mouse optic nerve following prenatal exposure to alcohol. J Anat 1997;191:49-56.

5. Pinazo-Duran MD, Renau-Piqueras J, Guerri C. Developmental changes in the optic nerve related to ethanol consumption in pregnant rats: analysis of the ethanol-exposed optic nerve. Teratology 1993;48:305-22.

6. Strömland K, Pinazo-Duran MD. Optic nerve hypoplasia: comparative effects in children and rats exposed to alcohol during pregnancy. Teratology 1994;50: 100-11.

7. Phillips DE. Effects of limited postnatal ethanol exposure on the development of myelin and nerve fibers in rat optic nerve. Exp Neurol 1989;103:90-100.

8. Phillips DE, Krueger SK. Effects of postnatal ethanol exposure on glial cell development in rat optic nerve. Exp Neurol 1990;107:97-105.

9. Harris SJ, Wilce P, Bedi KS. Exposure of rats to a high but not low dose of ethanol during early postnatal life increases the rate of loss of optic nerve axons and decreases the rate of myelination. J Anat 2000;197:477-85.

10. Kjellström C, Conradi NG. Decreased axonal calibres without axonal loss in optic nerve following chronic alcohol feeding in adult rats: a morphometric study. Acta Neuropathol (Berl) 1993;85:117-21.

11. Lescaudron L, Jaffard R, Verna A. Modifications in number and morphology of dendritic spines resulting from chronic ethanol consumption and withdrawal: a Golgi study in the mouse anterior and posterior hippocampus. Exp Neurol 1989;106:156-63.

12. Tavares MA, Paula-Barbosa MM. Alcohol-induced granule cell loss in the cerebellar cortex of the adult rat. Exp Neurol 1982;78:574-82.

13. Fukuda Y, Sugimoto T, Shirokawa T. Strain differences in quantitative analysis of the rat optic nerve. Exp Neurol 1982;75:525-32.

14. Walker DW, Barnes DE, Zornetzer SF, Hunter BE, Kubanis P. Neuronal loss in hippocampus induced by prolonged ethanol consumption in rats. Science 1980;209:711-3.

15. Phillips SC, Cragg BG. Chronic consumption of alcohol by adult mice: effect on hippocampal cells and synapses. Exp Neurol 1983;80:218-26. 